\title{
Bericht über das Kolloquium
}

\section{Vom Wort zum Text in der DaF-Didaktik, Pisa 13. 05. 2011}

Am 3. Mai 2011 fand in Dipartimento di Linguistica der Universität Pisa unter der wissenschaftlichen Leitung von Prof. Dr. Marina Foschi und Prof. Dr. Marianne Hepp das Kolloquium „Vom Wort zum Text in der DaF-Didaktik“ statt. Das Kolloquium wurde schon zum zweiten Mal im Rahmen der Zusammenarbeit zwischen Dipartimento di Linguistica der Universität in Pisa (Italien) und des Instituts für Angewandte Linguistik der Adam-Mickiewicz-Universität in Posen (Polen) realisiert.

Das Treffen eröffnete der Leiter des Promotionsstudiengangs Linguistik Prof. Dr. Franco Fanciullo. Nach der Begrüßung der Gäste übernahm Prof. Dr. Marina Foschi die Moderation der ersten Sektion des Kolloquiums. Zwei Gastvorträge rahmten die Tagung ein. In dem Eröffungsvortrag „Vergangenheitstempora im Deutschen und Italienischen“ sprach Dr. Nicole Schumacher (Berlin) über lexikalische und grammatische Mittel zum Ausdruck von Temporalität und Aspektualität im Deutschen und im Italienischen. Im ersten Teil der Vormittagssektion lag der Fokus auf der Kompetenz: Dr. Sabrina Ballestracci (Florenz) stellte in dem Vortrag „Sprachliche Kompetenz und übersetzerische Bewusstheit im DaF-Unterricht" die Ergebnisse des empirischen Versuchs dar. Dr. Agnieszka Błażek (Posen) thematisierte dagegen in ihrem Vortrag die Probleme bei der „Evaluation der interkulturellen Kompetenz im DaF-Bereich“. 
Nach einer kurzen Kaffeepause stellte Mag. Marc Träbert (Pisa) den Vortrag der abwesenden Referentin Dr. Valentina Dolfi (Pisa) dar: „Sprachmittel im Vergleich: Wie versöhnen sich die unterschiedlichen Grammatischen Ansätze im DAF-Unterricht?" Als zweite Referentin nach der Pause sprach Dr. Luiza Ciepielewska-Kaczmarek. Sie ging in ihrem Vortrag auf die Gruppe der jungen Lernenden ein und suchte eine Antwort auf die Frage, ob „Schreiben im frühen DaF-Unterricht eine zu Recht vernachlässigte Fertigkeit?" darstellt. Im Referat von Mag. Katharina Salzmann (Pisa) unter dem Titel „Zur Begründung meiner Wahl ..." lag der Fokus auf der Konstruktion der wissenschaftlichen Argumentation in Abschlussarbeiten italienischer Studierender.

Die Tagung wurde nach der Mittagspause fortgesetzt. Die Moderation der zweiten Sektion übernahm Prof. Dr. Marianne Hepp, die das Wort an Dr. Aldona Sopata (Posen) übergab. Die Referentin stellte die Theorie und Praxis zum „Literatureinsatz beim frühen Fremdsprachenerwerb“ dar. Auch im zweiten Referat der Nachmittagssektion bildeten die Kinder den Schwerpunkt: In ihrem Vortrag verdeutlichte Dr. Daniela Sorrentino (Modena) „Die Vermittlung juristischen Fachwissens in deutschsprachigen Kinderzeitungen am Beispiel des Umgangs mit der Nominalphrase“. Dr. Carolina Flinz (Pisa) machte dagegen zum Thema ihres Referats „Mikrostrukturelle Eigenschaften touristischer Fachtexte" und zog zum Vergleich deutsche und italienische Reisekataloge heran.

Bei Dr. Serena Carlini (Pisa) stand das Wörterbuch im Mittelpunkt. Die Referentin stellte die „DaF-Lerner als kundige Wörterbuchbenutzer" dar und überlegte in diesem Zusammenhang die Konsequenzen: "eine didaktische oder lexikographische Frage?" Dr. Patrizio Malloggi (Pisa) ging auf „Die Brainstorming-Methode als nicht traditioneller Einstieg in das Grammatikkapitel der Präpositionen bei lokalen Ausdrücken“ ein.

Prof. Dr. Erwin Tschirner (Universität Leipzig) hielt den Abschlussvortrag „Zur Progression von Präfix und Partikelverben: eine korpuslinguistische Untersuchung“.

Dank der an jeden Vortrag anschließenden Diskussion wurde das Kolloquium zum Forum eines regen Gedankenaustauschs und gab somit vielen Teilnehmern weitere Anstöße zur wissenschaftlichen Arbeit. Da die Referate als Sonderheft der Online-Zeitschrift des Dipartimento di Linguistica erscheinen, werden sie für alle interessierten Leser zum Nachlesen zugänglich sein. Schon heute kann die Lektüre empfohlen werden. 